\title{
Germination and vigour of seed of sorghum (Sorghum bicolor L. Moench) treated with Arsenicum album
}

\author{
Rosimar Maria Marques, Bruno Reis, Anielen Cassia Tormena Cavazin, \\ Flávia Carolina Moreira, Monica Buchoski, Hingrid Ariane Silva, \\ Mayara Lolis, Carlos Moacir Bonato
}

State University of Maringá (UEM), Maringá, PR, Brazil

\begin{abstract}
Introduction: The accelerated aging test evaluates the behavior of seeds exposed to stress conditions based on the fact that the rate of deterioration is considerably increased by exposure to high temperature and relative humidity [1]. The knowledge acquired in homeopathic science is experimental research on healthy living organisms that are undergoing experimentation using homeopathic medicines [2]. The experimental research on homeopathic plants has been performed in Europe, Mexico, India and Brazil, proving that the principle of homeopathy, as set out in the animal kingdom has been found in plants [3]. This experiment aimed to evaluate the effect of the homeopathic Arsenicum album in decimal (x) and centesimal (cH) scale on the physiological variables of the germination and growth of sorghum (Sorghum bicolor L.) subjected to accelerated aging.
\end{abstract}

Materials and methods: The experiment was conducted at the Laboratory of Plant Physiology and Homeopathy, Universidade Estadual de Maringá - UEM. Arsenicum album 6x and 2CH matrices were acquired in homeopathic pharmacy. From matrices were prepared further dynamizations in decimal (x) and centesimal (C) scale, according to the Brazilian Homeopathic Pharmacopoeia [4]. In the accelerated aging test were used samples of 200 seeds per treatment distributed evenly forming a single layer on the surfaces of metal wire suspended inside the plastic box containing inside $40 \mathrm{ml}$ of Arsenicum album (9, 12, 18, or 30x 24 and $9,12,18,24$ or 30C). The control consisted of distilled water. The boxes were placed in BOD-chamber adjusted to $42^{\circ} \mathrm{C}$ and relative humidity $100 \%$ for 96 hours. After this period, led to the germination test, using four replicates of 50 seeds, totalizing 52 experimental units which were placed on germination paper previously soaked with distilled water in an amount equivalent to 2.5 times the paper weight[5]. The rolls were arranged randomly in the BOD-chamber at $25^{\circ} \mathrm{C} \pm 2$. The interpretation of the test was performed after seven days [6]. Germination percentage (\% PG) was determined according to the Rules for Seed Analysis [5]. The length of primary roots (PRL) and hypocotyl (LH) were only performed in normal seedlings. The total length of the radicle (TLR) was calculated as the sum of the lengths of hypocotyl and primary root. The experimental design was completely randomized. We adopted the double-blind procedure, avoiding possible interference from the researcher. Data were subjected to analysis of variance and means compared by ScottKnott test $(\mathrm{P} \leq 0.05)$.

Results: Arsenicum album 9x, 9CH and $12 \mathrm{CH}$ reduced the percentage of germination (\%PG) (Figure 1A). The highest values in the length of hypocotyl (LH) were observed in 12 and $24 \mathrm{x}$ dilutions, followed by a reduction in intermediate and higher inhibition at 30x and $9 \mathrm{x}$. The $18 \mathrm{x}$ does not differ from control. All centesimal dilution presented effect on the variable analyzed. There was an increase in hypocotyl length in the 12, 18 and 24 dilutions, and reduction in 9 and $30 \mathrm{cH}$ dilutions (Figure 1B). The primary root length (PRL) of sorghum was increased in dilutions 18 and 30x. However, the centesimal dilutions were not different from control 
(Figure 1C). There was an increase in the total length of the seedlings (TLS) in the dilutions 18, 24 and 30x and reduction in $9 \mathrm{x}$ when compared to control. As for centesimal dilutions, there was an effect only in $18 \mathrm{cH}$. The other centesimal dilutions were not different from control (Figure 1D).
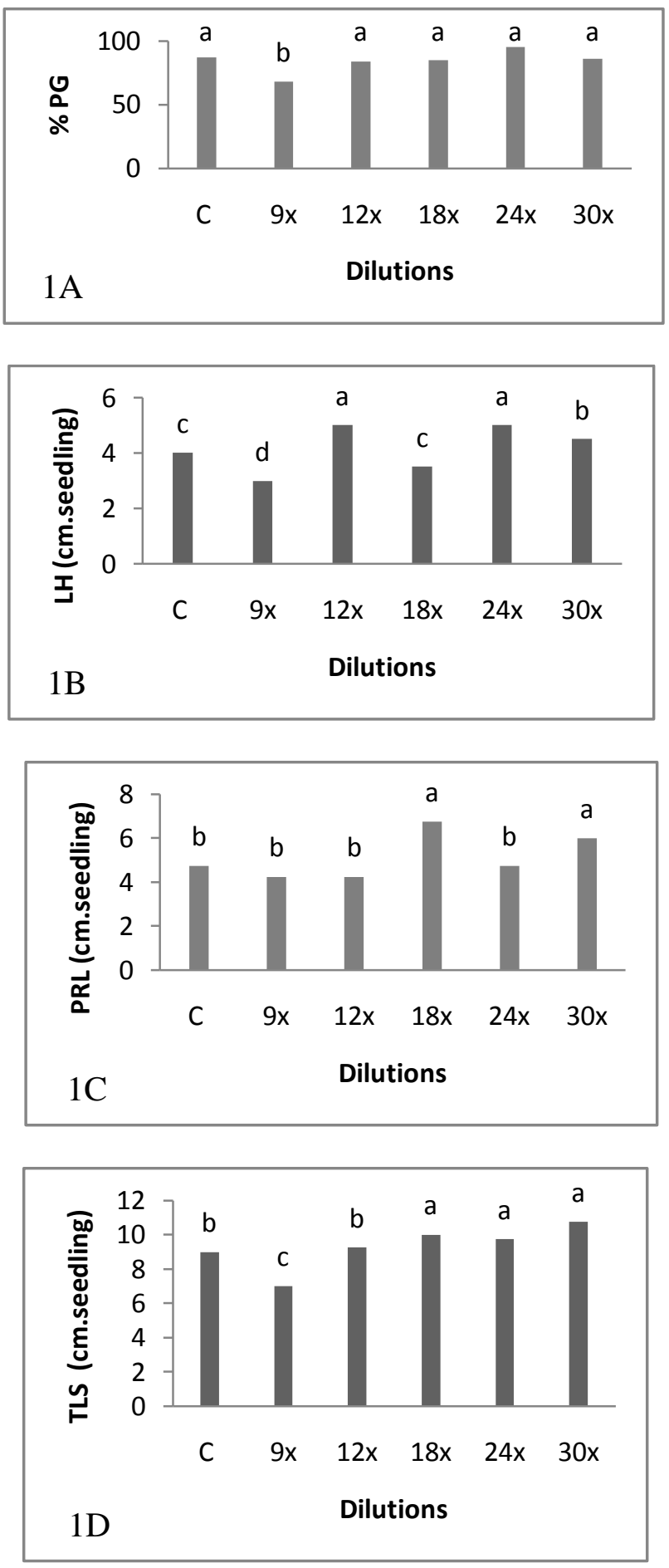
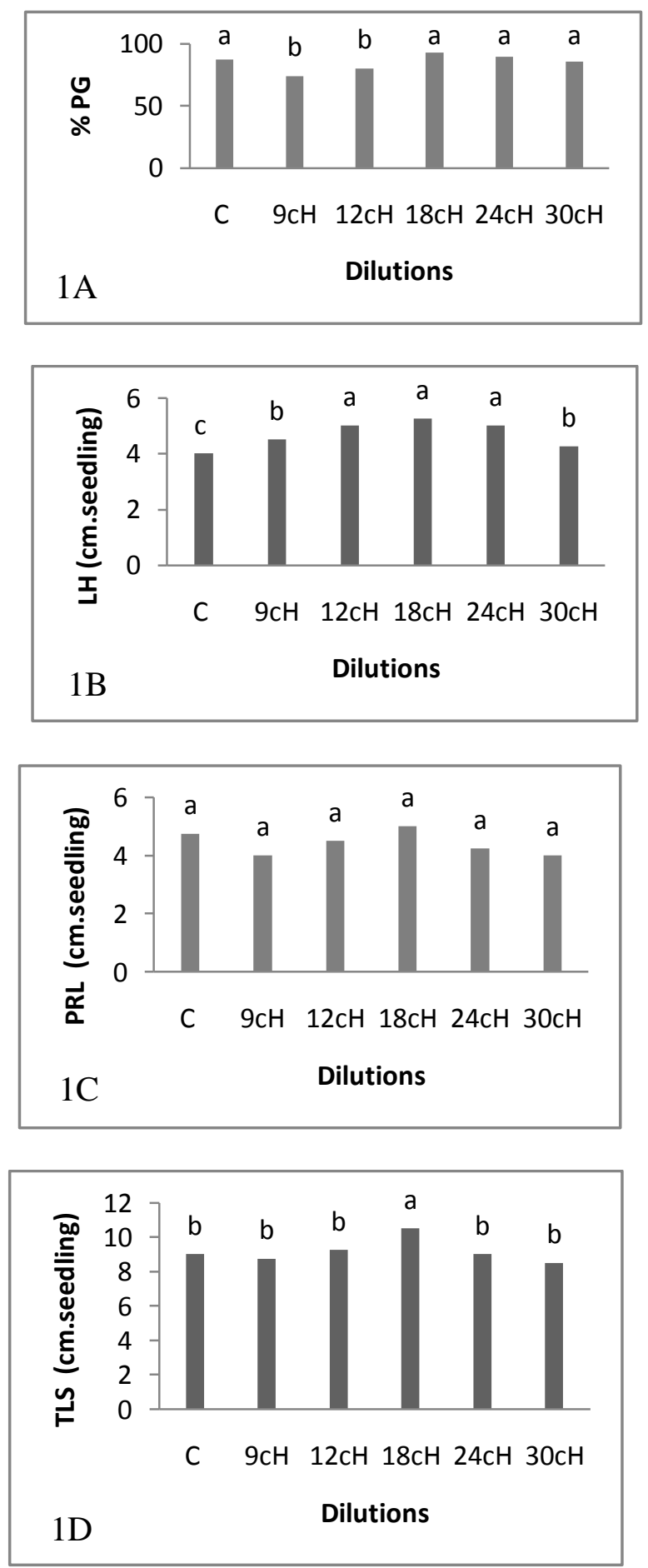

Figure 1. Effect of Arsenicum album in decimal (x) and centesimal (cH) scale on germination percentage (1A), hypocotyl length (1B) in primary root length (1C) and the total length seedlings (1D) of sorghum seeds subjected to accelerated aging. Means followed by same letter do not differ by Scott-Knott $(\mathrm{P}>0.05)$. 
Discussion: The accelerated aging consists of evaluating seed response, after they have been subjected to high temperature and relative humidity near $100 \%$ for some period of exposure. The seeds exposed to accelerated aging suffer serious degenerative changes in metabolism resulting in increase respiratory activity and reserves consumption causing a decrease in germination [1]. Similar effects in reducing germination percentage were found by Lahnstein et al. [7] in wheat seeds stressed with arsenic trioxide A. album 45dH. However, we can not say that the germination reduction in dilutions $9 \mathrm{x}, 9 \mathrm{cH}$ and $12 \mathrm{cH}$ resulted from direct action of $A$. album, or it is a consequence of the increase in abnormal seedlings in these dilutions. Vigorous seeds present more capacity to convert in storage tissues and the greater incorporation of these substances in embryonic axis resulting in a higher growth [1]. The increase in hypocotyl elongation in the dilutions 12 e $24 \mathrm{x}$ and 12, 18 and $24 \mathrm{cH}$ suggest more efficient use of reserves by seeds stressed. The increase in primary root length is a very important feature in the competition for nutrients and water in the soil. Manifestations of increase in length of the root system mediated by the same dilutions of homeopathic remedies were observed in decimal scale. The increase in the total seedlings of sorghum caused by dilution 18, 24 and $30 \mathrm{x}$ and $18 \mathrm{cH}$ showed the effectiveness of the dilutions in the stimulation of early growth of seedlings which is important in the early stages of development, it includes the establishment period of culture [6].

Conclusions: In general, homeopathic preparations did not affect the germination percentage except for 9x, and $9 \mathrm{cH} 12 \mathrm{cH}$ dilution. Furthermore, the dilutions reduced the effects on premature aging verified by high values of growth variables. These results suggest that high dilution Arsenicum album can be an alternative to minimize the deleterious effect to high temperature.

\section{References}

[1] Marcos Filho J. Fisiologia de sementes de plantas cultivadas. Fealq, 459 p, 2005

[2] Duarte ESM, Casali VWD, Cecon PR, Reis EL, Arruda VM, Moreira AM, Lisboa SP, Armond C, Silva CV. Isoterápicos no crescimento de mentrasto (Ageratum conyzoides L.). In: Seminário Brasileiro Sobre Homeopatia Na Agropecuária Orgânica, 2005, Nova Venécia-ES p. 175-185 [Português].

[3] Bonato CM. Homeopatia em Modelos Vegetais. Cultura Homeopática. 2007; n. 21, p.24-28.

[4] Brasil. Farmacopéia Homeopática Brasileira. 4 ed. São Paulo: Atheneu, 1997.

[5] Brasil. Ministério da Agricultura, Pecuária e Abastecimento. Regras para Análise de Sementes. Brasília: MAPA/ACS, 2009.

[6] Nakagawa J. Testes de vigor baseados na avaliação das plântulas. In: Vieira RD, Carvalho NM. (Ed.). Testes de vigor em sementes. Jaboticabal: Funep, 1994.

[7] Lahnstein L, Binder M, Thurneysen A, Frei-erb M, Betti L, Peruzzi M, Heusser P, Baumgartner S. Isopathic treatment effects of Arsenicum album 45x on wheat seedling growth - further reproduction trials. Homeopathy. 2009, 98, 198-207 [Inglês]. 


\title{
Germinação e vigor de sementes de sorgo forrageiro (Sorghum bicolor L.) tratadas com Arsenicum album
}

\begin{abstract}
Introdução: $\mathrm{O}$ teste de envelhecimento acelerado avalia o comportamento das sementes quando submetidas a condições de estresse, com base no fato de que a taxa de deterioração das sementes é aumentada consideravelmente pela exposição à temperatura e a umidade relativa altas [1].O conhecimento na ciência homeopática é adquirido com investigação experimental em organismos vivos sadios que são submetidos à experimentação utilizando medicamentos homeopáticos [2]. A investigação experimental homeopática em plantas tem sido executada na Europa, México, Índia e Brasil, comprovando que o princípio da homeopatia, consagrado no reino animal, tem sido verificado nos vegetais, a partir de respostas aos estímulos homeopáticos [3]. Este experimento teve por objetivo avaliar o efeito do medicamento homeopático Arsenicum album na escala decimal (x) e centesimal $(\mathrm{cH})$ sobre as variáveis fisiológicas da germinação e crescimento de sorgo (Sorghum bicolor L.) submetido ao envelhecimento acelerado.
\end{abstract}

Material e métodos: $\mathrm{O}$ experimento foi conduzido no Laboratório de Fisiologia e Homeopatia Vegetal da Universidade Estadual de Maringá - UEM. As matrizes homeopáticas Arsenicum album 6x e Arsenicum album $2 \mathrm{cH}$, foram adquiridas em farmácia homeopática idônea. A partir das matrizes foram preparadas as demais dinamizações na escala decimal (x) e centesimal $(\mathrm{cH})$, de acordo com as instruções contidas na Farmacopéia Homeopática Brasileira [4]. No teste de envelhecimento acelerado foram utilizadas amostras de 200 sementes para cada tratamento distribuídas uniformemente, de maneira a formar uma camada simples sobre a superfície da tela metálica suspensa no interior da caixa tipo gerbox, contendo no seu interior $40 \mathrm{~mL}$ dos tratamentos Arsenicum album (9, 12, 18, 24 e 30x e 9, 12, 18, 24 e 30cH) . O controle constituiu-se de água destilada. As caixas foram colocadas em câmara tipo BOD, ajustada a $42^{\circ} \mathrm{C}$ e $100 \%$ de umidade relativa, durante 96 horas. Após esse período, conduziu-se o teste de germinação, utilizando-se 4 repetições de 50 sementes, totalizando 52 unidades experimentais as quais foram distribuídas em papel de germinação previamente umedecido com água destilada, em quantidade equivalente a 2,5 vezes o peso do papel [5]. Em seguida, confeccionaram-se rolos os quais foram dispostas aleatoriamente na câmara de germinação tipo BOD a temperatura de $25^{\circ} \mathrm{C} \pm 2$. A interpretação do teste foi realizada após sete dias [6]. Os critérios de porcentagens de germinação (PG \%) foram feitos de acordo com as recomendações das Regras para Análise de Sementes [5]. Os comprimentos da raiz primária (CRP) e hipocótilo (CHP) foram efetuados somente para plântulas normais. O comprimento total da plântula (CTP) foi calculado pela soma dos comprimentos do hipocótilo e da raiz primária. O delineamento experimental foi inteiramente casualizado. Foi adotado o procedimento duplo-cego, evitando-se possíveis interferências ou tendências do pesquisador. Os dados foram submetidos à análise de variância e as médias comparadas pelo teste Scott-Knott $(\mathrm{P} \leq 0.05)$.

Resultados: Os tratamentos interferiram significativamente na porcentagem de germinação (PG \%). A diluição 9x, 9cH e 12cH de A. album reduziram a porcentagem de germinação (Figura 1A). Em relação ao comprimento do hipocótilo (CHP) das plântulas de sorgo, os maiores valores foram observados nas diluições decimais 12 e 24x, seguido de uma redução intermediária na 30x e maior inibição na 9x. A 18x não diferiu do controle. Todas as diluições centesimais causaram efeito na variável analisada. Observou-se incremento no comprimento do hipocótilo nas diluições 12, 18 e $24 \mathrm{cH}$ e redução nas dinamizações 9 e 30cH (Figura 1B). O comprimento da raiz primária (CRP) das plântulas de sorgo apresentou incremento nas diluições 18 e 30x. Entretanto, as diluições centesimais não diferiram do controle (Figura 1C). Observou-se que houve incremento no comprimento total da plântula (CTP) nas diluições 18, 24 e 30x e redução na 9x quando comparada ao 
controle. Quanto às diluições centesimais, verificou-se efeito somente na $18 \mathrm{cH}$. As demais diluições centesimais não diferiram do controle (Figura 1D).
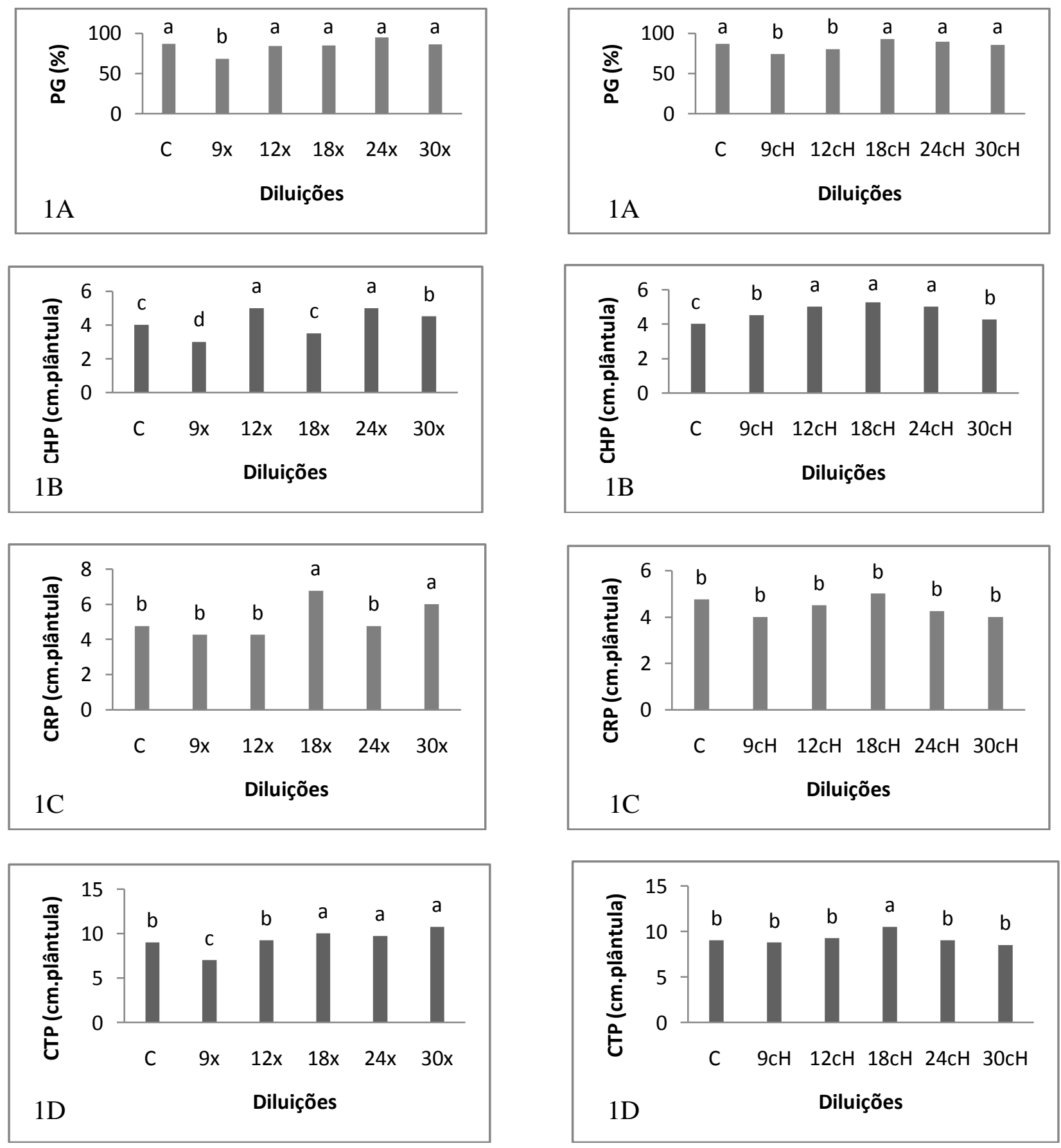

Figura 1. Efeito das diluições decimais (x) e centesimais $(\mathrm{cH})$ do medicamento homeopático Arsenicum album na porcentagem de germinação (A), no comprimento do hipocótilo (B), no comprimento da raiz primária (C) e no comprimento total da plântula (D) de sementes de sorgo submetidas ao envelhecimento acelerado. Médias seguidas de mesma letra não diferem entre si pelo teste Scott-Knott $(\mathrm{P}>0.05)$. 
Discussões: O teste de envelhecimento acelerado consiste em avaliar a resposta das sementes, após elas terem sido submetidas à temperatura elevada e umidade relativa do ar próxima a $100 \%$, por determinado período de exposição. As sementes quando submetidas ao envelhecimento acelerado sofrem sérias alterações degenerativas no metabolismo resultando em intensa atividade respiratória e consumo de reservas, causando decréscimo na germinação [1]. Efeitos similares na redução da \% GER foram encontrados por Lahnstein et al. [7], em sementes de trigo estressadas com trióxido de arsênico, tratadas com o medicamento homeopático $A$. album 45DH. Contudo, não se pode afirmar que a redução da porcentagem de germinação nas diluições 9x, $9 \mathrm{cH}$ e $12 \mathrm{cH}$ foi resultante da ação direta de A. album, ou uma conseqüência do aumento de plântulas anormais nestas diluições. Sementes vigorosas apresentam maior capacidade de transformação das reservas nos tecidos de armazenamento e maior incorporação dessas pelo eixo embrionário resultando maior crescimento [1]. O incremento no alongamento do hipocótilo nas diluições 12 e $24 \mathrm{x}$ e 12,18 e $24 \mathrm{cH}$ pode ser um índice sensível da eficiência na utilização das reservas pelas sementes estressadas. $\mathrm{O}$ aumento no comprimento da raiz primária é uma característica muito importante na competição por nutrientes e água no solo. Manifestações de incremento do comprimento do sistema radicular mediados por diluições de um mesmo medicamento homeopático foram observadas somente na escala decimal. O incremento no comprimento total das plântulas de sorgo provocado pelas diluições 18, 24 e 30x e 18cH evidenciam a eficácia das diluições no estimulo do crescimento precoce das plântulas, sendo este importante nos estádios iniciais do desenvolvimento, pois compreende o período de estabelecimento da cultura [6].

Conclusões: Em geral, as preparações homeopáticas não afetaram a porcentagem de germinação, exceto para 9x, e diluição $12 \mathrm{CH}$ e $9 \mathrm{CH}$. Além disso, as diluições reduziram os efeitos do envelhecimento prematuro verificada por altos valores nas variáveis de crescimento. Estes resultados sugerem que altas diluições de Arsenicum album podem ser uma alternativa para minimizar o efeito deletério à alta temperatura.

\section{(cc) EY-No-ND Licensed to GIRI}

Support: authors declare that this study received no funding

Conflict of interest: authors declare there is no conflict of interest

Correspondence author: Carlos Moacir Bonato, cmbonato@uem.br; cmbonato@hotmail.com

How to cite this article: Marques RM, Reis B, Cavazin ACT, Moreita FC, Buchoski MG, Sinva HA, Lois MA, Bonato CM. Germination and vigour of seed of sorghum (Sorghum bicolor L. Moench) treated with Arsenicum album. Int J High Dilution Res [online]. 2011 [cited YYYY Month dd]; 10(36): 239-244. Proceedings of the XXV GIRI Symposium and VIII CBFH; 2011 Sep 04-07; Foz do Iguaçu (Brazil). GIRI and ABFH; 2011; Available from: http://www.feg.unesp.br/ ojs/index.php/ijhdr/article/view/507/518 